\title{
The expression of Homo Sapiens microRNA-21 (Hsa-miR-21-5p) and mRNA reversion inducing cysteine rich protein with kazal motifs (RECK) in plasma of epithelial ovarian cancer
}

\author{
Kartika $\mathrm{Al}^{1 *}$, Chasanah $\mathrm{SN}^{2}$, Fitriawan $\mathrm{AS}^{2}$, Tanjung DS ${ }^{1}$, Fitria MS ${ }^{1}$, Pukan $\mathrm{FK}^{2}$, \\ Oktriani $\mathrm{R}^{3}$, Trirahmanto $\mathrm{A}^{4}$, Pradjatmo $\mathrm{H}^{4}$, Aryandono $\mathrm{T}^{5}$, Haryana $\mathrm{SM}^{6}$. \\ ${ }^{1}$ Post Graduate Program in Biotechnology, School of Postgraduate, Universitas \\ Gadjah Mada, Yogyakarta. ${ }^{2}$ Post Graduate Program in Biomedical Sciences, \\ Faculty of Medicine, Universitas Gadjah Mada, Yogyakarta. ${ }^{3}$ Department of \\ Biochemistry, Faculty of Medicine, Universitas Gadjah Mada Yogyakarta. \\ ${ }^{4}$ Department of Obstetry Gynecology, Faculty of Medicine, Universitas Gadjah \\ Mada, Yogyakarta. ${ }^{5}$ Department of Surgery, Faculty of Medicine, Universitas \\ Gadjah Mada, Yogyakarta. ${ }^{6}$ Department of Histology and Cellular Biology, Faculty \\ of Medicine, Universitas Gadjah Mada, Yogyakarta
}

DOI: http://dx.doi.org/10.19106/JMedScieSup0048042016015

\section{ABSTRACT}

Epithelial Ovarian Cancer (EOC) is a malignant cancer with high mortality among Indonesian women. EOC showed no specific symptoms in its early stages, thus making the screening mostly occur when patients are in advanced stage. Treatment of advance-stage EOC is more challenging and prognosis is poor. Therefore, minimally-invasive biomarkers are needed to diagnose at the early stage. microRNA is one of the potential biomarkers which not only expressed inside the cell, but also secreted outside the cell with exosome protection. This protection makes microRNA stable. Moreover, several studies have shown the ability to detect microRNA in the blood sample. microRNA-21 (miR-21) is oncomiR targeting tumor suppressor mRNA RECK based on in-silico analysis.

The first aim of this study is to determine the expression of miR-21 in plasma samples of EOC patients compared to healthy controls. The second aim is to investigate the expression correlation between miR-21 and RECK mRNA.

Blood samples were collected from 30 patients and 30 healthy controls. Plasma was then obtained from centrifuged blood samples. The total RNA was isolated and reverse transcribed to produce cDNAs. cDNAs were then quantified using qPCR using specific primer for miR-21 and RECK mRNA. The expression analysis 
was done using relative expression method by Livak. The expression of miR-21 was calculated using the miR-16 expression as the reference gene. Also, Betaactin was used as reference gene for RECK mRNA calculation. The correlation between the expression of miR-21 and mRNA RECK was analyzed using the Spearman rho correlation analysis.

This study revealed that the expression of miR-21 in EOC patients increased 4.7579-fold compared to healthy controls ( $p<0.05$ ). On the other hand, the miR-21 target, RECK mRNA, decreased 4,2 times with fold change 0.237665 on plasma of EOC patients compared to healthy controls $(p<0.05)$. The statistical calculation of the expression of miR-21 and mRNA RECK was inversely proportional to mRNA RECK with a strong correlation.

This study was able to prove that the expression of miR-21 is upregulated in EOC patients and confirmed by the downregulation of RECK expression. The future research to design anti-miR-21 and suppress the expression of miR-21 in EOC is required, and analysis of the effect of miR-21 in the development of EOC might also be performed.

Keywords: Hsa-miR-21-5p, EOC, plasma microRNA, mRNA RECK, microRNA expression in cancer and healthy. 